\title{
The External Genitalia Score (EGS): A \\ European multicenter validation study
}

Saskia van der Straaten ${ }^{1}$, MD, Alexander Springer ${ }^{2}, \mathrm{MD}, \mathrm{PhD}$, Aleksandra Zecic ${ }^{3}$, MD, Doris Hebenstreit $^{4}$, MD, Ursula Tonnhofer ${ }^{2}$, MD, Aneta Gawlik ${ }^{5}, \mathrm{MD}, \mathrm{PhD}$, Malgorzata Baumert ${ }^{6}$, MD, PhD, Kamila Szeliga ${ }^{5}$, MD, Sara Debulpaep ${ }^{7}$, MD, An Desloovere ${ }^{7}$, Lloyd Tack ${ }^{1}$, MD, Koen Smets ${ }^{3}, \mathrm{MD}, \mathrm{PhD}$, Malgorzata Wasniewska ${ }^{8}, \mathrm{MD}, \mathrm{PhD}$, Domenico Corica ${ }^{8}, \mathrm{MD}$, Mariarosa Calafiore ${ }^{8}$, MD, Marie Lindhardt Ljubicic ${ }^{9}$, MD, Alexander Siegfried Busch ${ }^{9}$, MD, Anders Juul $^{9}$, MD, PhD, Anna Nordenström ${ }^{10}$, MD, PhD, Jon Sigurdsson ${ }^{10}$, MD, Christa E. Flück $^{11}$, MD, PhD, Tanja Haamberg ${ }^{11}$, MD, Stefanie Graf ${ }^{11}$, MD, Sabine E Hannema ${ }^{12,13}$, MD, PhD, Katja P. Wolffenbuttel ${ }^{14}$, MD, Olaf Hiort ${ }^{15}$, MD, PhD, S. Faisal Ahmed ${ }^{16}$, MD, FRCPCH , Martine Cools ${ }^{1}, \mathrm{MD}, \mathrm{PhD}$

${ }^{1}$ Ghent University Hospital, Department of Pediatrics, Division of Pediatric Endocrinology and Ghent University, Department of Internal Medicine and Pediatrics, Ghent, Belgium

${ }^{2}$ Medical University of Vienna, Department of Pediatric Surgery, Vienna, Austria

${ }^{3}$ Ghent University Hospital, Department of Neonatology, Ghent, Belgium

${ }^{4}$ Medical University Innsbruck, Department of Urology

${ }^{5}$ Medical University of Silesia, Department of Pediatrics, Pediatric Endocrinology, Katowice, Poland

${ }^{6}$ Medical University of Silesia, Department of Neonatology, Katowice, Poland

${ }^{7}$ Ghent University Hospital, Department of Pediatrics, Ghent, Belgium

${ }^{8}$ University Hospital of Messina, Department of Human Pathology of Adulthood and Childhood, Messina, Italy

${ }^{9}$ Copenhagen University Hospital - Rigshospitalet, Department of Growth and Reproduction, Copenhagen, Denmark

${ }^{10}$ Karolinska University Hospital Stockholm, Department of Women's and Children's Health Karolinska Institutet, Stockholm, Sweden

${ }^{11}$ Bern University Children's Hospital Inselspital, Department of Pediatrics, Division of Pediatric Endocrinology, Diabetology and Metabolism and Department of BioMedical Research, University of Bern, Bern, Switzerland

${ }^{12}$ Erasmus MC - Sophia Children's Hospital, University Medical Center Rotterdam,

Department of Pediatric Endocrinology, Rotterdam, the Netherlands

${ }^{13}$ Leiden University Medical Centre, Department of Paediatrics, Leiden, The Netherlands

${ }^{14}$ Erasmus MC - Sophia Children's Hospital, University Medical Center Rotterdam,

Department of Urology and Paediatric Urology, Rotterdam, the Netherlands

${ }^{15}$ University of Lübeck, Division of Paediatric Endocrinology and Diabetes, Department of Paediatrics, Lübeck, Germany

${ }^{16}$ Developmental Endocrinology Research Group, University of Glasgow, Glasgow, UK

Keywords: 'External Genitalia Score', 'External Masculinization Score', anogenital distances, atypical genitalia

Corresponding author's contact information : Martine Cools, Ghent University Hospital, Princess Elisabeth Childrens' Hospital, Building 3K12D, room 319, Corneel Heymanslaan 10, 9000 Ghent, Belgium, martine.cools@ugent.be

(C) Endocrine Society 2019. All rights reserved. For permissions, please e-mail: journals.permissions@ oup.com. jc.2019-01437. See endocrine.org/publications for Accepted Manuscript disclaimer and additional information. 
Grants, funding source supporting the writing of this paper: This article is based upon work from COST Action DSDnet, supported by COST (European Cooperation in Science and Technology), grant BM1303. MC holds a senior clinical investigator grant from the Research Foundation Flanders, AG, KS and MB are supported by statutory work of Medical University of Silesia (number KNW-1-011/N/8/K). M.L.L, A.S.B and A.J are supported by COPENHAGEN Minipuberty Study (R146-A5644) and The Danish Environmental Protection Agency and Aase and Ejnar Danielsens Foundation (10-00101874). CEF is supported by the Swiss National Science Foundation (grant number 320030-146127).

Disclosure summary: The authors have indicated that they have no financial relationships relevant to this article to disclose. All authors have no conflict of interest relevant to this article to disclose.

\section{Contributors' Statement Page}

Saskia van der Straaten coordinated overall data collection, performed the interobserver validation study, performed local data collection, analysed results and drafted the manuscript. Alexander Springer developed the EGS, designed the study, supervised the interobserver validation study, coordinated local data collection, analysed results and revised the manuscript.

Aleksandra Zecic supervised local data collection and revised the manuscript.

Doris Hebenstreit performed the interobserver validation study, performed local data collection, and revised the manuscript.

Ursula Tonnhofer, Aneta Gawlik, Malgorzata Baumert, Kamila Szeliga, Sara Debulpaep, An Desloovere, Lloyd Tack, Koen Smets, Malgorzata Wasniewska, Domenico Corica, Mariarosa Calafiore, Marie Lindhardt Ljubicic, Alexander Siegfried Busch, Anders Juul, Jon Sigurdsson, Tanja Haamberg, Stefanie Graf, Sabine E. Hannema and Katja P. Wolffenbuttel performed local data collection, took part in analyses of the results and revised the manuscript.

Anna Nordenström and Christa E. Flück developed the EGS, designed the study, coordinated local data collection, analysed results and revised the manuscript.

Olaf Hiort coordinated the COST Action, supervised the overall project and revised the manuscript.

S. Faisal Ahmed developed the EGS, analysed results and revised the manuscript.

Martine Cools developed the EGS, designed the study, performed local data collection, analysed results, drafted the manuscript and supervised the overall project.

All authors approved the final manuscript as submitted and agree to be accountable for all aspects of the work. 


\section{Abbreviations}

AGDs - Anogenital distances

AGDac - Ano-clitoral distance from the centre of the anus to the anterior base of the clitoris AGDaf - Ano-fourchettal distance from the centre of the anus to the fourchette

AGDap - Ano-penile distance from the centre of the anus to the anterior base of the penis AGDas - Ano-scrotal distance from the centre of the anus to the posterior base of the scrotum

AGDlower - measured from the centre of the anus to the base of the labio/scrotal border AGDupper - measured from the centre of the anus to the anterior base of the genital tubercle

AGDl/u - lower / upper AGD ratio

COST - European Cooperation in Science and Technology

EGS - External Genitalia Score

PS - Prader Score

EMS - External Masculinization Score

DSDs - Differences of sex development

ICC - Interclass correlation coefficient

$\mathrm{CI}$ - confidence interval 


\begin{abstract}
Context: Standardized description of external genitalia is needed in the assessment of children with atypical genitalia.
\end{abstract}

Objectives: To validate the External Genitalia Score (EGS), to present reference values for preterm and term babies up to 24 months and correlate obtained scores with anogenital distances (AGDs).

Design, Setting: A European multicentre $(n=8)$ validation study was conducted from 07/2016 until 07/2018.

\title{
Patients and Methods
}

EGS is based on the external masculinization score but uses a gradual scale from female to male (range 0-12) and terminology appropriate for both sexes. The reliability of EGS and AGD's were determined by the interclass correlation coefficient (ICC). Cross-sectional data were obtained in 686 term (0-24 months), and 181 preterm babies and 111 babies with atypical genitalia.

Results: ICC of EGS in typical and atypical genitalia is excellent and good. Median EGS $\left(10^{\text {th }}-90^{\text {th }}\right.$ centile) in males $<28$ weeks gestation is $10(8.6-11.5)$; in males $28-32$ weeks 11.5 (9.2-12); in males 33-36 weeks 11.5 (10.5-12) and in full-term males 12 (10.5-12). In all female babies, EGS is $0(0-0)$. The mean (SD) AGDl/u is $0.45(0.1)$, with significant difference between AGDl/u in males $0.49(0.1)$ and females $0.39(0.1)$ and in-between values in DSD 0.43 (0.1). AGDl/u correlates with EGS in males with typical genitalia and in atypical genitalia.

Conclusions: EGS is a reliable and valid tool to describe external genitalia in premature and term babies up to 24 months. EGS correlates with AGDl/u in males. It facilitates standardized assessment, clinical decision-making and multicenter research.

\section{Précis}

The EGS is a new instrument to describe external genitalia, enabling comprehensive assessment of atypical genitalia. The EGS was validated and reference values for preterm and term babies up to 24 months are presented. 


\section{Introduction}

Differences (or disorders) of sex development (DSDs) are heterogeneous congenital conditions that affect the development of the urogenital tract and reproductive system and result in atypical sex differentiation. ${ }^{1-4}$ The incidence of DSDs where sex assignment may be unclear at birth is estimated at $1 / 5500$ births. ${ }^{5}$ For milder variations such as hypospadias, prevalence rates vary from 13.8 to $40 / 10.000 .{ }^{6-7}$ The clinical management of these conditions is complex and requires specialised care by a multidisciplinary team. ${ }^{1,8}$ A precise understanding of the underlying cause, preferably up to the molecular genetic level, is crucial to allow individualized management as well as for research purposes. Detailed evaluation of the genital phenotype will inform clinicians about the need for further referral to an expert center, and guide them to specific diagnostic tests such as hormonal, imaging and genetic investigations. ${ }^{9}$ The genital phenotype at birth has also been related to long-term outcomes, e.g. with regard to genital (dis)satisfaction ${ }^{10}$, the prevalence of cardiac ${ }^{11}$ or other comorbidities ${ }^{12}$ or risk for the development of gonadal germ cell tumors. ${ }^{13,14}$ The relevance of a precise description of the genital phenotype has even increased in recent years as genital surgery in childhood has become controversial, and many children who have a DSD nowadays grow up with a genital difference. The long-term outcome of this approach will need to be determined. Lastly, given that the individual DSD conditions are (very) rare, meaningful research requires a multicenter approach and thus a standardized battery of tools across centers to assess and document this phenotypic variability.

A comprehensive genital exam contains the following landmarks: the presence and location of the gonads, genital tubercle development, degree of fusion of the labio-scrotal folds and location of the urethral meatus. A micropenis is defined as a short penis, $\leq 24-25 \mathrm{~mm}$, i.e. $\leq$ 2.5 SD below the mean and with a normal configuration. ${ }^{15}$ Minor racial differences for SPL have been published. ${ }^{16}$ The distance between the anus and various landmarks of the external 
genitalia has been shown to be a sensitive index of androgen activity during fetal development and is sexually dimorphic. ${ }^{17,18}$ Various anogenital distances (AGDs) have been proposed. In male term newborns, the mean (SD) anoscrotal anogenital distance (AGDas), measured from the centre of the anus to the posterior scrotal wall is 24.7 (4.5) $\mathrm{mm}$. In female term newborns the mean (SD) anofourchette AGD (AGDaf), measured from the centre of the anus to the fourchette is 16.0 (3.2) mm. ${ }^{17}$ AGDas and AGDaf are represented in Figure 1 as lower AGD (AGDl); anopenile AGD (AGDap) and anoclitoral AGD (AGDac) are represented as upper AGD (AGDu). A shorter AGDas and penile length have been found in infants with hypospadias and cryptorchidism, a longer AGDaf has been described in female infants with androgen excess, e.g. in congenital adrenal hyperplasia (CAH). In typical female infants, it was shown that calculating the anogenital-ratio (AGDaf/ac) offers advantages as it follows a normal distribution and does not correlate with anthropometric variables or gestational age. 19,20 The Prader score (PS) was developed by Andrea Prader in 1954 to capture genital variation in children who have CAH. Apart from the typical female and male phenotypes, it categorizes external genitalia in children with $\mathrm{CAH}$ in 5 additional stages with progressive virilization from a phenotypic female with mild clitoromegaly (stage 1) to a phenotypic male with glandular hypospadias (stage 5). ${ }^{21}$ In 2000, the External Masculinization Score (EMS) was introduced to improve the initial assessment of boys with a genital difference. The EMS (range 0-12) allocates points to five different characteristics of the external genitalia (scrotal

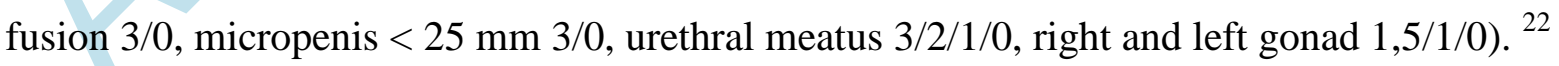
The EMS allows standardization of genital assessment, but a refinement of the score is needed to capture the appearance of the genitalia more comprehensively across the phenotypic spectrum in both sexes. We here present the External Genitalia Score (EGS) (Table 1 and supplemental Table $1^{23}$ ) as a modified, non-binary version of EMS. EGS was developed by Working Group 1 of the European Cooperation in Science and Technology 
(COST) Action BM1303. The EGS uses a gradual scale from female to male (range 0-12) of the same anatomical landmarks as the EMS. To provide a full description of the external genitalia, the various AGDs were measured and, in line with EGS, a gender-neutral lower / upper AGD ratio (AGDl/u) was calculated as a marker of genital virilisation independent of body weight (Figure 1).

\section{Materials and Methods}

Based on expert opinion and group discussions, members of the DSDnet COST Action (www.dsdnet.eu) working group 1 modified the existing EMS to describe the same anatomical features with a refined categorical scale for the items labio/scrotal fusion, urethral meatus and the position of the gonads and a continuous scale for the size of the genital tubercle, ranging from typical female to typical male (Table 1 and supplemental Table $1^{23}$ ). In addition, the vocabulary was adjusted in a way that suits both sexes.

\section{Measurements}

Genital assessment and measurements included EMS and EGS, PS, and AGD's. The same digital caliper (Carbon Fiber Digital Caliper, resolution: 0.1 mm, QST-Express, type QST008, China) was used for all measurements across centers. Length of the genital tubercle (GTL) was measured along its dorsal aspect in a non-erect state, gently stretching it between two fingers until the point of increased resistance, from the base of the genital tubercle (as close to the pubic bone as possible) to the tip of the glans and excluding the foreskin. ${ }^{15}$ The measurement was performed twice, and the mean was calculated. Location of the gonads was determined by palpation, as described by Ogilvy-Stuart. ${ }^{3}$ The position of the meatus and degree of labio-scrotal fusion were determined by visual inspection. AGD measurements were standardized according to the Infant Development and the Environment Study (TIDES) ${ }^{17}$, with some modifications, and the accompanying training video (kindly provided by the 
TIDES research group) was distributed among participating centers. Modifications to TIDES method: The baby is placed in the middle of the bed instead of at the edge to allow the same position in premature babies in the incubator. For the same reason, the fixed end of the caliper is held at the centre of the anus, and the sliding part of the caliper is moved while measuring the AGD's. The sliding part is touching but not compressing the skin at the anterior base of the genital tubercle to standardize measurements in male and female infants. The examiner does not use a marker for the mid-anus position but chooses a wrinkle in the centre of the anus to use for the measurement of the two AGD's. The average of two measurements is used for analysis instead of the average of three measurements. AGDl was measured from the centre of the anus to the base of the labio/scrotal border and AGDu was measured from the centre of the anus to the anterior base of the genital tubercle. PS was determined by visual inspection and EMS, EGS and AGDl/u were calculated based on the obtained scores and AGD measurements.

\section{Participants}

First, the inter-observer reliability of PS, EMS, EGS and inter- and intra-observer reliability of AGDl/u were determined by two observers from two different centers in 35 babies with typical genitalia (12 female, 23 male; 12 preterm, 23 term). Subsequently, the reliability of these parameters was assessed by two observers in four different centers in 66 babies with atypical genitalia (males with "mild non-specific undermasculinization"; i.e. isolated hypospadias $(n=29)$ or isolated cryptorchidism (n=8)”, 46, XY DSD (n=22), Sex Chromosome DSD (n=2) and 46,XX DSD (n=5) (Supplemental Table 2) ${ }^{23}$. A collaborative multicenter study was then conducted in eight European clinical centers from July 2016 until July 2018, to establish reference data for the EGS in typical genitalia (Table 2 ). For this purpose, the external genitalia of preterm infants, term infants up to 1 month and babies from 1 to 24 months were assessed by one observer per center and PS, EMS, EGS, 
GTL and AGDl/u were determined. The following potential covariates were noted: maternal age, ethnicity, virilization and medication use in pregnancy, exposure to toxic products, smoking in pregnancy, history of consanguinity, gestational age at birth, weight and length at birth, weight and length at assessment. Children with a major congenital malformation (central, cardiac, pneumologic, urologic) were excluded. In total, 105 male and 76 female preterm (<37 weeks) neonates, 178 male and 200 female term neonates, and 153 male and 155 female babies aged 1-24 months were assessed. In four clinical centers the PS, EMS, EGS and AGD 1/u were obtained in babies with atypical genitalia (see Supplemental Table 3 for participant characteristics ${ }^{23}$ ).

\section{Statistical analyses}

The inter-observer reliability of the PS, EMS and EGS and the intra- and inter-observer reliability for AGDs were assessed by Intraclass Correlation Coefficient (ICC) estimates and their $95 \%$ confidence intervals (CI), based on absolute-agreement, 2-way random-effect model. ${ }^{24}$ The median $\left(10^{\text {th }}-90^{\text {th }}\right.$ centile $)$ for EGS and EMS were generated. Spearman's rho determined the correlation between EGS and EMS as both have a skewed distribution. The Bland-Altman analysis was used to assess agreement between the EGS and EMS. This method calculates the mean difference between two methods, and 95\% limits (2 SD) of agreement of the differences between the two methods. ${ }^{25}$ The mean $\left(\mathrm{SD}, 10^{\text {th }}-90^{\text {th }}\right.$ centile $)$ for the different AGDs and AGD-ratios was calculated in typical and in atypical genitalia. Correlations of the different AGDs and the AGDl/u with weight, length and age were assessed by Pearson analysis. Potential covariates of the different AGDs and AGDl/u were assessed by linear regression. A Spearman's correlation was done to determine the relationship between EGS and AGDl/u. An independent-samples t-test was conducted to compare AGDl/u in typical and atypical genitalia. All analyses were performed using the SPSS statistical package version 25 . 


\section{Ethics}

The study was approved by the local ethical committees of each participating center (Local IDs: Ghent: B670201628499, Medical University of Vienna 1872/2014), Rotterdam: MEC2016-706, Copenhagen: H-15014876 and RH-2015-210-04146, Katowice:

KNW/0022/KB1/158/I/16/17/18, Stockholm Karolinska University Hospital 2008/167-31/3, 2009-01-13, 10-12-16. Messina: MEC 104/16. Informed consent was obtained from at least one parent or legal guardian for each child.

\section{Results}

Inter-observer reliability of EGS in comparison with EMS, PS and of the various AGDs

As the EGS is a more refined modification of the EMS, we compared its reliability to the original EMS, and to the PS, which is historically the most widely used. Inter-observer ICC $(n=35)$ for EGS showed no case of disagreement between any scorer (excellent) in typical genitalia $(\mathrm{ICC}=1)$ and, the interobserver variability in atypical genitalia $(\mathrm{n}=66)$ was good (ICC=0.89, CI 0.82-0.93). Likewise, inter-observer ICC for PS and EMS also showed no case of disagreement in typical and were moderate and good in atypical genitalia. Interobserver ICC for the different AGDs and genital tubercle length were moderate for AGDu and good for AGDl and genital tubercle length in typical male genitalia and good for AGDu, AGDl and genital tubercle length in atypical genitalia. Inter-observer ICC were good for AGDl and, AGDu in typical female genitalia. Intra-observer ICC for the different AGDs and genital tubercle length were good or excellent in both typical and atypical genitalia (Supplemental Table 2) ${ }^{23}$.

\section{Reference data for genital tubercle length, EGS, AGDs and AGDI/u}

As a new measuring instrument, we established reference data for EGS, including data in preand dysmature babies who present more often with atypical genitalia. ${ }^{12}$ In addition, we 
determined AGDs and AGDl/u to investigate correlations of EGS with other measures of genital virilisation. In male term infants with typical genitalia, the mean (SD) genital tubercle length ( $\mathrm{n}=174)$, AGDl and AGDu (n=178) were $31.2(5.4), 24.6(4.7)$ and $47.6(5.8) \mathrm{mm}$ respectively. In female term infants with typical genitalia $(n=200)$, the mean (SD) length of AGDl and AGDu were 14.8 (3.5) and 37.8 (4.5) mm respectively. AGDl/u was independent of body weight (Figure 2). Although mean (SD) AGDl/u in male infants [0.49(0.1)], significantly differs from AGDl/u in female infants [0.39 (0.1)], large overlap exists between both groups (Table 3, Figure 2). AGD 1/u in male neonate positively correlates with gestational age $(\mathrm{r}(243)=0.3, \mathrm{p}<0.05)$. No univariate or bivariate correlation was detected between AGD 1/u and any of the other covariates (maternal age, ethnicity, center, virilization and/or medications used in pregnancy, exposure to toxic products or smoking during pregnancy). In typical male infants, the median and $10^{\text {th }}$ centile EGS gradually rise with increasing gestational age and birth weight due to increasing genital tubercle length and descent of the testes (Figure $3 \mathrm{~A}$ and $3 \mathrm{~B}$ ). In addition, the EGS $10^{\text {th }}$ centile gradually increases with age up to 24 months. Median EGS in typical female premature and full-term babies up to 24 months is $0(0-0)$ (Table 2).

\section{Genital tubercle length, EGS, AGDs and AGDl/u in children with atypical external genitalia}

In babies with atypical genitalia, the EGS covers the whole phenotypic spectrum, resulting in scores ranging from 0 to 12 with large overlap between the various DSD categories $(46, \mathrm{XX}$ DSD, 46,XY DSD and 45,X/46,XY DSD) (Table 4 and Figure 3C). In male babies with atypical genitalia (46,XY DSD and "mild non-specific undermasculinization”), AGDl/u $(\mathrm{M}=0.43, \mathrm{SD}=0.11)$ is significantly shorter than $\mathrm{AGDl} / \mathrm{u}$ in typical males $(\mathrm{M}=0.49$, $\mathrm{SD}=0.09) ; \mathrm{t}(95.1)=4.8, \mathrm{p}<0.05)$, however AGDl/u widely varies in babies with atypical 
genitalia, with a mean 0.43 (0.1 SD) not different from mean AGDl/u $0.45(0.1)$ in babies with typical genitalia (Figure 2).

\section{Correlation and agreement between scores and measures}

AGDl, AGDu and AGDl/u positively correlate with EGS in typical male full term neonates as well as in babies with atypical genital phenotypes $\left(\mathrm{r}_{\mathrm{s}}(243)=0.19, \mathrm{p}<0.05\right.$ and $\mathrm{r}_{\mathrm{s}}(78)=0.35$, $\mathrm{p}<0.05$ respectively) (Supplemental Table 4$)^{23}$. As expected, there is a strong, positive correlation between EGS and EMS in typical $\left(\mathrm{r}_{\mathrm{s}}(853)=0.97, \mathrm{p}<0.05\right)$ and atypical genitalia $\left(\mathrm{r}_{\mathrm{s}}(110)=0.9, \mathrm{p}<0.05\right)($ Supplemental Figure $1 \mathrm{~A}){ }^{23}$. The Bland-Altman analysis shows that optimal agreement between the two methods is reached for EMS/EGS results $<3$ and $>9.5$ (Supplemental Figure 1B) ${ }^{23}$.

\section{Discussion}

The EMS, developed by Ahmed et al. in $2000^{22}$ provides an objective and standardized tool to describe external genitalia in male babies and has been correlated with various DSD-related outcomes. ${ }^{10-14}$ A major limitation of the EMS in the work-up of an infant with atypical genitalia is that it cannot be applied in assigned females because of the gender-specific design and vocabulary (e.g. micropenis yes/no, scrotal fusion yes/no). Also, EMS does not capture the full phenotypic spectrum of genital variation that characterises DSD conditions due to its dichotomous nature. To overcome these problems, COST Action BM1303 working group 1 modified the EMS in a gender-neutral and more refined categorical scale, that better reflects the naturally occurring variation (e.g. by introducing the option "posterior labioscrotal fusion"). The resulting tool was termed the EGS and was subsequently validated in a large European multicenter study. EGS can be applied in both typical male and female babies and in babies who have variations in their genital characteristics. We provide normative data for premature, low birth weight and full-term babies until the age of two years for a mixed 
European population. Such data are of particular relevance given the frequent association in males of intra-uterine growth retardation with genital undermasculinization and the difficulties in assessing genital variation in preterm infants whose testes have not yet descended and whose penis has not yet reached its full-term length. Although the EGS can be used for the initial evaluation of babies with atypical genitalia, it cannot fully replace a more detailed qualitative genital description. The EGS does not inform on the presence of other atypical genital features such as complete or partial penoscrotal transposition, scrotal anomalies or degree of penile curvature. Moreover, EGS, like EMS, does not provide information on important internal genital characteristics in the context of DSD, such as the presence of a urogenital sinus or the location of the vaginal confluence in $46, \mathrm{XX}$ babies who have CAH. Bland-Altman analysis reveals that EGS and EMS have least agreement in the group of children with atypical genitalia, i.e. children who have an EGS between 3 and 9.5. In our data of 66 children with a DSD, the IQRs are smaller for EGS as compared to EMS, support our hypothesis that the EGS allows a more refined description of genital virilisation. In addition, EGS is easy to use, helps to assess important landmarks of the external genitalia, also by physicians who do not examine a baby with variant genital development on a regular basis and, it is an attractive alternative for genital photography, which has ethical constraints. Due to its objectivity and simple design, it is also very instrumental for the exchange of data on genital phenotypes between centers and researchers, for example through large-scale registries such as I-DSD. Future research and clinical use of EGS will reveal if specific EGS outcomes can be allocated to specific diagnoses/mutated genes, but based on our preliminary data, it is expected that EGS will have little predictive value regarding the underlying diagnosis in most cases, given the large overlap between the various DSD categories. Reference data for the EGS in term, preterm and low birthweight children, are of high relevance for a broad audience of paediatricians and general practitioners. According to 
Ahmed et al ${ }^{9}$, clinical evaluation by a specialized DSD-team is advised in proximal forms of hypospadias, isolated micropenis, isolated clitoromegaly, any form of familial hypospadias, and those who have a combination of genital variations resulting in an EMS of less than 11. All these variations will result in a maximal EGS of 10.5, corresponding to P10 in full-term male infants. Therefore, based on our data, we advise referral to a specialised DSD team of any full-term infant who has an EGS $>0$ and $\leq 10.5$ (or $\leq$ P10), and of any preterm or low birthweight infant who has an EGS $>0$ and $\leq$ P10 for gestational age or birthweight, independent of maternal age, ethnicity, virilization and/or medications used in pregnancy, exposure to toxic products or maternal smoking. Of note, the obtained EGS will not lead to a specific diagnosis in an infant who has variant genitalia, but it may justify further genetic, biochemical and hormonal diagnostic investigations. Further research is mandatory to determine if this recommendation will require adjustments in the future.

The anogenital distance has been shown a surrogate marker of prenatal androgen exposure and has been correlated to various endocrine-reproductive outcomes. ${ }^{26-28}$ Although it adds to the description of the external genitalia ${ }^{29}$, its clinical use is limited as it is relatively timeconsuming and measurements are hard to standardize among different observers. As AGD is known to correlate with anthropometric variables, which was confirmed in our study, the AGD-ratio may represent a more useful marker. In our study, AGD1/u followed a normal distribution and did not correlate with any of the anthropometric variables. Moreover, while mean AGDl/u significantly differs between typical males and typical females, this measure underscores the naturally encountered variation in genital phenotypes, both in typical males and females and in children who have a DSD, as becomes obvious from Figure 2. As expected, AGDl/u correlates with EGS in undermasculinized infants, both measurements independently reflecting the degree of prenatal androgen exposure. 
A major strength of our study is its multicenter design, allowing data collection in a large European sample in a relatively short period. At the same time, this multicenter approach may constitute a weakness as some measurements, such as AGD and genital tubercle length are prone to larger inter-observer variability. This was also confirmed by the variable ICC scores obtained for these measures in our study and this may explain the relatively large SD obtained for these parameters. In addition the assessment of children with atypical genitalia was performed in 4 out of 8 centers, which could have led to recruitment bias.

In conclusion the EGS is a reliable and easy-to-use tool that allows objective and detailed description of typical and variant external genitalia in neonates and infants. This facilitates clinical management and data exchange across centers, to study outcomes or draw genotypephenotype correlations. We here provide European reference data for term and premature neonates, for neonates who have low birthweight and for toddlers up to 24 months.

\section{Acknowledgements}

The authors would like to thank Mrs Ellen Deschepper for statistical support.

\section{Data Availability}

The datasets generated during and/or analysed during the current study are not publicly available but are available from the corresponding author on reasonable request. 


\section{References}

1. Cools M, Nordenstrom A, Robeva R, Hall J, Westerveld P, Fluck C, Kohler B, Berra M, Springer A, Schweizer K, Pasterski V. Caring for individuals with a difference of sex development (DSD): a Consensus Statement. Nature reviews Endocrinology. 2018.

2. Lee PA, Nordenstrom A, Houk CP, Ahmed SF, Auchus R, Baratz A, Baratz Dalke K, Liao LM, LinSu K, Looijenga LH, 3rd, Mazur T, Meyer-Bahlburg HF, Mouriquand P, Quigley CA, Sandberg DE, Vilain E, Witchel S. Global Disorders of Sex Development Update since 2006: Perceptions, Approach and Care. Horm Res Paediatr. 2016;85(3):158-180.

3. Ogilvy-Stuart AL, Brain CE. Early assessment of ambiguous genitalia. Archives of disease in childhood. 2004;89(5):401-407.

4. Hughes IA, Houk C, Ahmed SF, Lee PA. Consensus statement on management of intersex disorders. Archives of disease in childhood. 2006;91(7):554-563.

5. Thyen U, Lanz K, Holterhus PM, Hiort O. Epidemiology and initial management of ambiguous genitalia at birth in Germany. Hormone research. 2006;66(4):195-203.

6. Loane M, Dolk H, Kelly A, Teljeur C, Greenlees R, Densem J. Paper 4: EUROCAT statistical monitoring: identification and investigation of ten year trends of congenital anomalies in Europe. Birth Defects Res A Clin Mol Teratol. 2011;91 Suppl 1:S31-43.

7. Springer A, van den Heijkant M, Baumann S. Worldwide prevalence of hypospadias. Journal of pediatric urology. 2016;12(3):152.e151-157.

8. Hiort O, Birnbaum W, Marshall L, Wunsch L, Werner R, Schroder T, Dohnert U, Holterhus PM. Management of disorders of sex development. Nature reviews Endocrinology. 2014;10(9):520-529.

9. Ahmed SF, Achermann JC, Arlt W, Balen A, Conway G, Edwards Z, Elford S, Hughes IA, Izatt L, Krone N, Miles H, O'Toole S, Perry L, Sanders C, Simmonds M, Watt A, Willis D. Society for Endocrinology UK guidance on the initial evaluation of an infant or an adolescent with a suspected disorder of sex development (Revised 2015). Clinical endocrinology. 2016;84(5):771-788.

10. van de Grift TC, Cohen-Kettenis PT, de Vries ALC, Kreukels BPC. Body image and self-esteem in disorders of sex development: A European multicenter study. Health Psychol. 2018;37(4):334-343.

11. De Groote K, Cools M, De Schepper J, Craen M, Francois I, Devos D, Carbonez K, Eyskens B, De Wolf $D$. Cardiovascular pathology in males and females with $45, \mathrm{X} / 46, \mathrm{XY}$ mosaicism. PLoS One. 2013;8(2):e54977.

12. Cox K, Bryce J, Jiang J, Rodie M, Sinnott R, Alkhawari M, Arlt W, Audi L, Balsamo A, Bertelloni S, Cools M, Darendeliler F, Drop S, Ellaithi M, Guran T, Hiort O, Holterhus PM, Hughes I, Krone N, Lisa L, Morel Y, Soder O, Wieacker P, Ahmed SF. Novel associations in disorders of sex development: findings from the I-DSD Registry. The Journal of clinical endocrinology and metabolism. 2014;99(2):E348-355.

13. Lindhardt Johansen M, Hagen CP, Rajpert-De Meyts E, Kjaergaard S, Petersen BL, Skakkebaek NE, Main KM, Juul A. 45,X/46,XY mosaicism: phenotypic characteristics, growth, and reproductive function--a retrospective longitudinal study. The Journal of clinical endocrinology and metabolism. 2012;97(8):E1540-1549.

14. Cools M, Pleskacova J, Stoop H, Hoebeke P, Van Laecke E, Drop SL, Lebl J, Oosterhuis JW, Looijenga LH, Wolffenbuttel KP. Gonadal pathology and tumor risk in relation to clinical characteristics in patients with $45, \mathrm{X} / 46, \mathrm{XY}$ mosaicism. The Journal of clinical endocrinology and metabolism. 2011;96(7):E1171-1180.

15. Lee PA, Mazur T, Danish R, Amrhein J, Blizzard RM, Money J, Migeon CJ. Micropenis. I. Criteria, etiologies and classification. The Johns Hopkins medical journal. 1980;146(4):156163.

16. Cheng PK, Chanoine JP. Should the definition of micropenis vary according to ethnicity? Hormone research. 2001;55(6):278-281. 
17. Sathyanarayana S, Grady R, Redmon JB, Ivicek K, Barrett E, Janssen S, Nguyen R, Swan SH. Anogenital distance and penile width measurements in The Infant Development and the Environment Study (TIDES): methods and predictors. Journal of pediatric urology. 2015;11(2):76.e71-76.

18. Thankamony A, Ong KK, Dunger DB, Acerini CL, Hughes IA. Anogenital distance from birth to 2 years: a population study. Environ Health Perspect. 2009;117(11):1786-1790.

19. Callegari C, Everett S, Ross M, Brasel JA. Anogenital ratio: measure of fetal virilization in premature and full-term newborn infants. The Journal of pediatrics. 1987;111(2):240-243.

20. Mondal R, Chatterjee K, Samanta M, Hazra A, Ray S, Sabui TK, Banerjee B, Das S, Roychowdhury D, Biswas R. Clitoral length and anogenital ratio in Indian newborn girls. Indian Pediatrics. 2016;53(4):299-303.

21. Prader A. Genital findings in the female pseudohermaphroditism of the congenital adrenogenital syndrome; morphology, frequency, development and heredity of the different genital forms. Helv Paediatr Acta. 1954;9:231-248 (1954).

22. Ahmed SF, Khwaja O, Hughes IA. The role of a clinical score in the assessment of ambiguous genitalia. BJU international. 2000;85(1):120-124.

23. van der Straaten S; Cools M. Supplemental figures and tables of European multicenter validation study of the External Genitalia Score. Dryad Digital Repository; 2019. https://doi.org/10.5061/dryad.sbcc2fr23.

24. Koo TK, Li MY. A Guideline of Selecting and Reporting Intraclass Correlation Coefficients for Reliability Research. J Chiropr Med. 2016;15(2):155-163.

25. Bland JM, Altman DG. Statistical methods for assessing agreement between two methods of clinical measurement. Lancet. 1986;1(8476):307-310.

26. Toppari J, Virtanen HE, Main KM, Skakkebaek NE. Cryptorchidism and hypospadias as a sign of testicular dysgenesis syndrome (TDS): environmental connection. Birth Defects Res A Clin Mol Teratol. 2010;88(10):910-919.

27. Eisenberg ML, Hsieh MH, Walters RC, Krasnow R, Lipshultz LI. The relationship between anogenital distance, fatherhood, and fertility in adult men. PLoS One. 2011;6(5):e18973.

28. Eisenberg ML, Jensen TK, Walters RC, Skakkebaek NE, Lipshultz LI. The relationship between anogenital distance and reproductive hormone levels in adult men. J Urol. 2012;187(2):594598.

29. Bangalore Krishna K, Houk CP, Lee PA. Pragmatic approach to intersex, including genital ambiguity, in the newborn. Seminars in perinatology. 2017;41(4):244-251. 
Figure 1. Measurement of Anogenital distances. In order to obtain a single measure that is suitable for all babies, AGDap and AGDac were defined as AGDu, and AGDas and AGDaf as AGDl.

Abbreviations:, AGDl (AGDlower): measured from the centre of the anus to the base of the labio/scrotal border, AGDu (AGDupper): measured from the centre of the anus to the anterior base of the genital tubercle

Figure 2. Correlation between $\mathrm{AGDl} / \mathrm{u}$ and weight in babies with typical genitalia and atypical genitalia

Abbreviations: ratio AGDl/u: lower/upper AGD ratio, AGDl: measured from the centre of the anus to the base of the labio/scrotal border, AGDu: measured from the centre of the anus to the anterior base of the genital tubercle

Figure 3. Boxplot with median and interquartile range of EGS (dark grey) in comparison with EMS (light grey). A: Results for typical male babies according to gestational age.

B: Results for typical male babies according to birthweight. C. Results for babies with atypical genitalia and various DSD groups.

Abbreviations: Mild non specific undermasculinization: refers to isolated hypospadias or isolated cryptorchidism

Table 1. "External Genitalia Score" describe phenotypic features at 5 anatomical landmarks of the genitalia: degree of labioscrotal fusion, length of the genital tubercle, position of the urethral meatus, and location of the right and left gonad. The final score is the sum of points allocated to feature 1-5.

Abbreviations: EGS: External Genitalia Score. GTL: genital tubercle length

Table 2. EGS in female and male babies with typical genital phenotypes in different gestational age, birthweight and age groups

Abbreviations: EGS: External Genitalia Score

Table 3. Genital tubercle length, AGDs and AGDl/u in male (light grey) and female (dark grey) babies with a typical genital phenotype.

Abbreviations: AGD: anogenital distance, AGDl/u: lower/upper AGD ratio, AGDu (AGDupper): measured from the centre of the anus to the anterior base of the genital tubercle, and AGDl (AGDlower): measured from the centre of the anus to the base of the labio/scrotal border.

Table 4. EGS median, $10^{\text {th }}-90^{\text {th }}$ centile scores and AGDl/u in babies with atypical genital phenotypes

Abbreviations:AGDu: measured from the centre of the anus to the anterior base of the genital tubercle, and AGDl: measured from the centre of the anus to the base of the labio/scrotal border. AGDl/u: lower/upper AGD ratio EGS: External Genitalia Score, * mild non-specific undermasculinization refers to males with isolated cryptorchidism or isolated hypospadias 

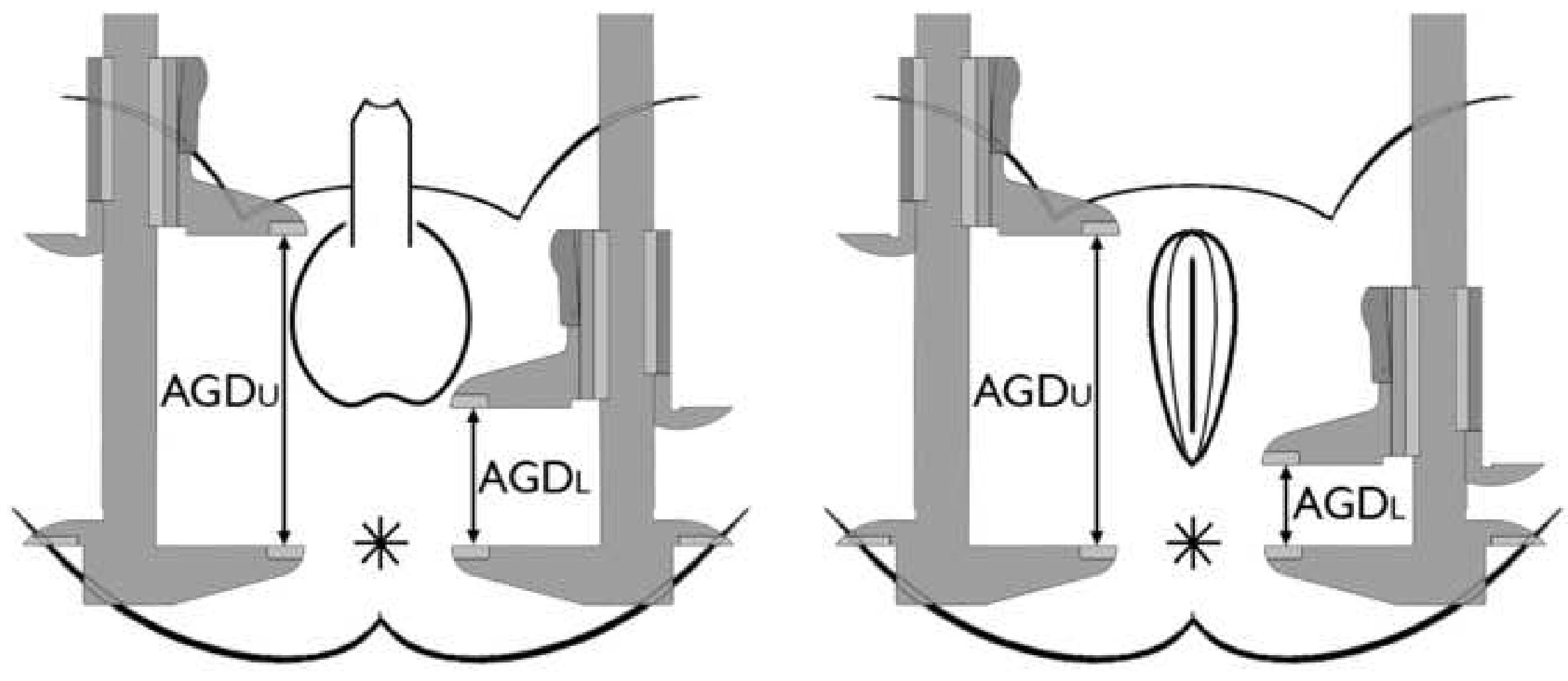


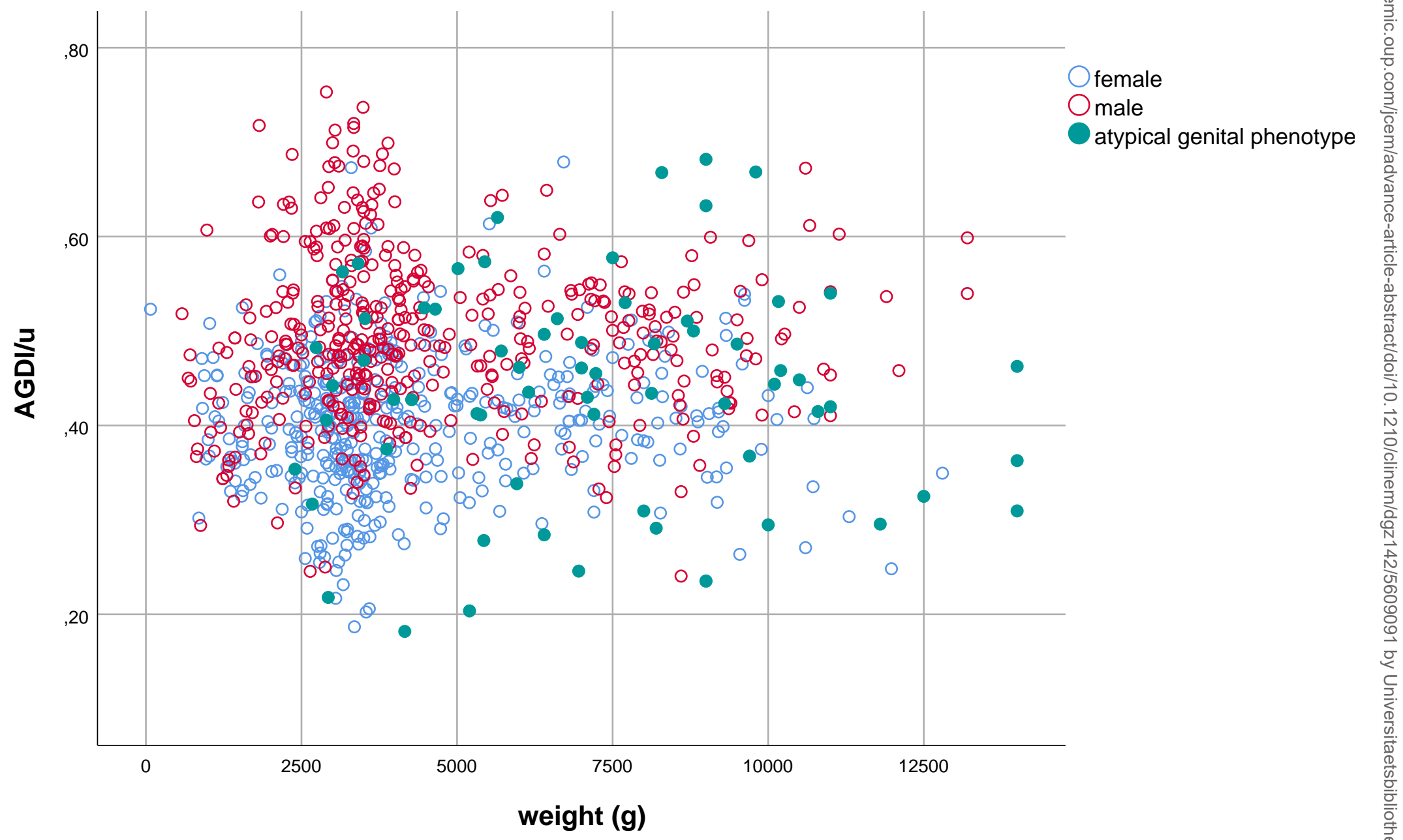



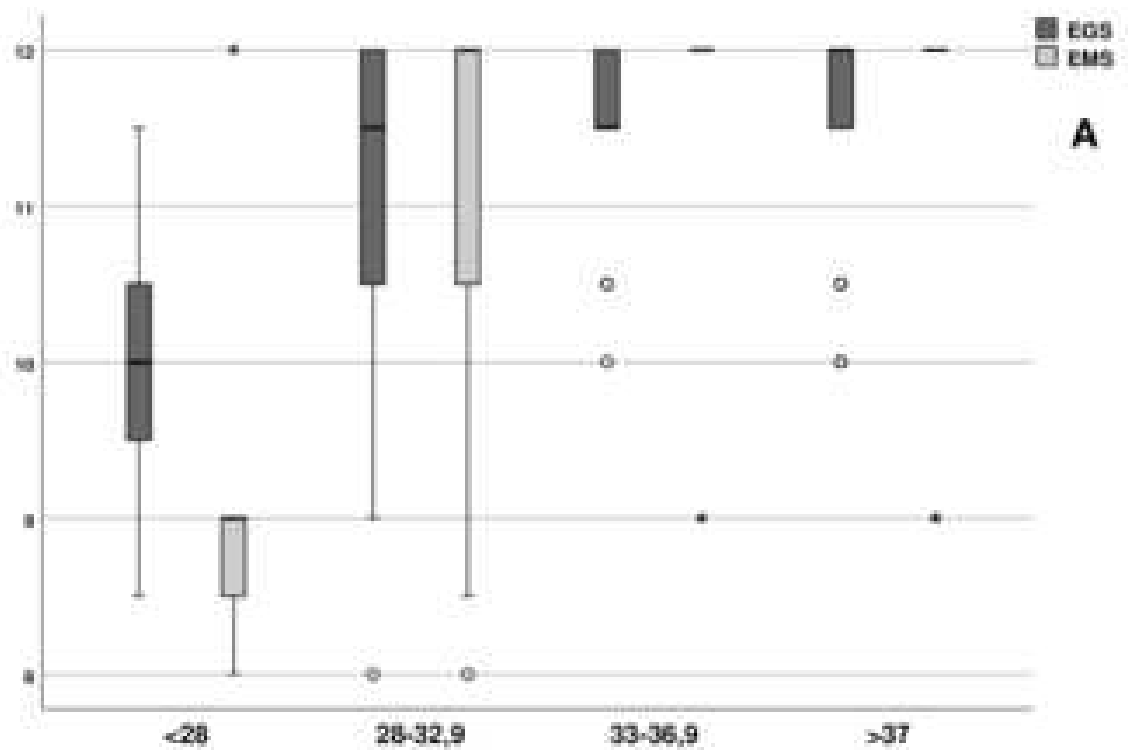

Gestational age (woeks)
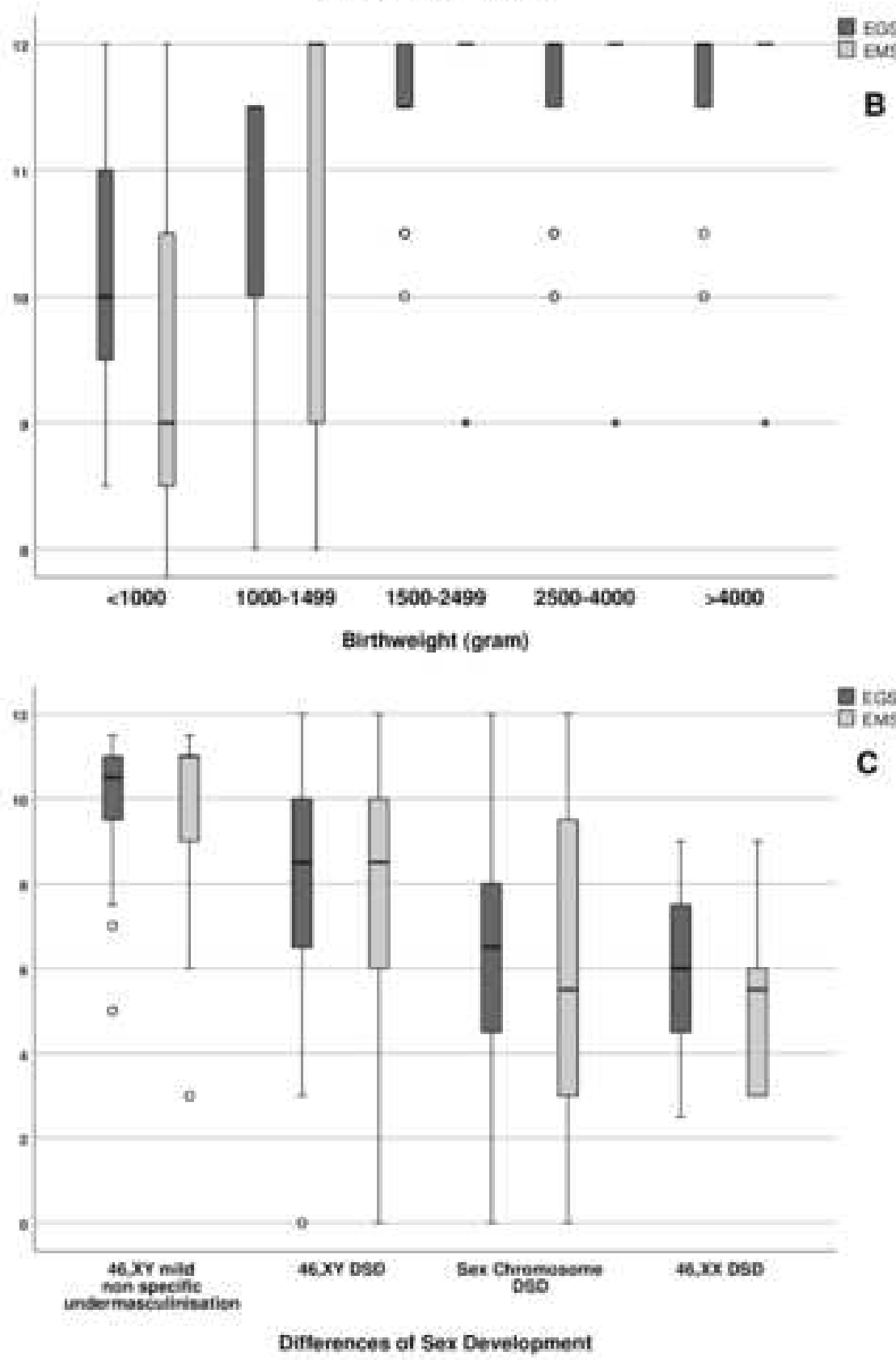